\title{
A 20-year experience with urgent percutaneous cardiopulmonary bypass for salvage of potential survivors of refractory cardiovascular collapse
}

\author{
Brian E. Jaski, MD, ${ }^{\mathrm{a}}$ Bryan Ortiz, ${ }^{\mathrm{b}}$ Koteswara R. Alla, ${ }^{\mathrm{b}}$ Sidney C Smith, Jr, MD, ${ }^{\mathrm{c}}$ Dale Glaser, PhD, ${ }^{\mathrm{b}}$ \\ Cynthia Walsh, MSN, ${ }^{\mathrm{b}}$ Suzanne Chillcott, BSN, RN, ${ }^{\mathrm{b}}$ Marcia Stahovich, RN, CCRN, ${ }^{\mathrm{b}}$ \\ Robert Adamson, $\mathrm{MD},{ }^{\mathrm{b}}$ and Walter Dembitsky, $\mathrm{MD}^{\mathrm{b}}$
}

\begin{abstract}
Objective: In-hospital cardiac arrest or refractory shock carries a high mortality despite the use of advanced resuscitative measures. We have implemented an in-hospital, nurse-based, continuously available, percutaneous, venoarterial cardiopulmonary bypass system, also known as extracorporeal life support (ECLS), as an adjunct to resuscitation when initial measures are ineffective.
\end{abstract}

\begin{abstract}
Methods: In 1986, a system for the rapid initiation of ECLS, was created in which trained critical care nurses primed an ECLS circuit and in-house physicians percutaneously placed required cannulas. From a prospective registry, we assessed long-term survival (LTS) ( $\geq 30$ days, cardiopulmonary support weaned), short-term survival ( $<30$ days, CPS weaned), or death on CPS.

Results: One hundred fifty patients (age, $57 \pm 17$ years) were urgently started on CPS for cardiac arrest ( $\mathrm{n}=127$; witnessed, $\mathrm{n}=124$; unwitnessed, $\mathrm{n}=3$ ) and refractory shock $(\mathrm{n}=23)$. Sixty-nine patients were weaned from CPS, and 81 could not be weaned. Overall, $39(26.0 \%)$ patients achieved LTS with a subsequent Kaplan-Meier median survival of 9.5 years. Duration of CPS was $32 \pm 38$ hours for LTS and $21 \pm 38$ hours for non-LTS. LTS occurred in $29(23.4 \%)$ of 124 patients started on CPS for witnessed cardiac arrest and $11(47.8 \%)$ of 23 for refractory shock $(P<.05)$. Among patients with CPS initiated in the cardiac catheterization laboratory, LTS was seen in $24(50.0 \%)$ of 48 versus $15(14.7 \%)$ of 102 in patients with CPS initiated in other locations $(P<.001)$. Cardiopulmonary resuscitation times greater than or equal to 30 minutes were associated with lower LTS $(P<.05)$. The most common cause of death during CPS was refractory cardiac dysfunction (39.5\%), and the most common cause associated with short-term survival was neurologic/pulmonary dysfunction (53.6\%). Seven patients were bridged to a left ventricular assist device, and 1 subsequently underwent heart transplantation. Multivariate analysis revealed only cardiac catheterization laboratory site of initiation as a significant independent predictor of LTS $(P<.01)$. When dividing the 20 -year experience in tertiles, recent recipients have had more common prearrest insertion. Rates of long-term survival have not changed.
\end{abstract}

Conclusion: Of patients started on CPS, $46 \%$ were weaned, and $26.0 \%$ were long-time survivors. Rapid initiation of CPS permits LTS for some inpatients with cardiovascular collapse when initial advanced resuscitation fails. Strategies to improve end-organ function associated with use of CPS should lead to greater LTS. This practical application of inexpensive available technology should be more widely used. (J Thorac Cardiovasc Surg 2010;139:753-7)

\section{Supplemental material is available online.}

Despite improvements in techniques of cardiopulmonary resuscitation (CPR), hospitalized patients who undergo

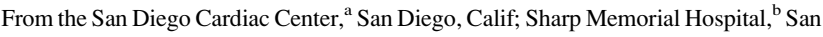
Diego, Calif; and the University of North Carolina, ${ }^{\mathrm{c}}$ Chapel Hill, NC.

Read at the Thirty-fifth Annual Meeting of The Western Thoracic Surgical Association, Banff, Alberta, Canada, June 24-27, 2009.

Disclosures: None.

This work was supported in part by a grant from the Azus Fund, Sharp Health Care Foundation.

Received for publication June 18, 2009; revisions received Oct 26, 2009; accepted for publication Nov 13, 2009.

Address for reprints: Brian E. Jaski, MD, San Diego Cardiac Center, 3131 Berger Ave, San Diego, CA 92123. (E-mail: bjaski@sdcardiac.com).

$0022-5223 / \$ 36.00$

Copyright (C) 2010 by The American Association for Thoracic Surgery

doi:10.1016/j.jtcvs.2009.11.018
}

refractory shock or cardiac arrest still demonstrate dismal rates of long-term survival. Using standardized criteria, ${ }^{1}$ a recent multicenter study in the United States found a survival to discharge after adult in-hospital cardiac arrest of $17 \% .^{2}$ In patients who require more than 10 minutes of CPR, a 30-day survival of $15.0 \%$ has been reported in a single-center report. ${ }^{3}$

In 1954, Gibbon ${ }^{4}$ reported the development of a cardiopulmonary bypass system that allowed surgeons to perform complex cardiac repairs for the first time. In 1985, a portable cardiopulmonary support (CPS) system was developed (C. R. Bard, Inc, Billerica, Mass) that could be used with percutaneous catheters, making it possible to implement cardiopulmonary bypass outside the operating room without opening the chest. ${ }^{4}$ The extracorporeal system was designed for rapid implementation in patients who would otherwise not survive with refractory cardiac or respiratory failure. 


$$
\begin{aligned}
& \text { Abbreviations and Acronyms } \\
& \begin{aligned}
\mathrm{CNS} & =\text { central nervous system } \\
\mathrm{CPR} & =\text { cardiopulmonary resuscitation } \\
\mathrm{CPS} & =\text { cardiopulmonary support } \\
\mathrm{LTS} & =\text { long-term survivor } \\
\mathrm{PEA} & =\text { pulseless electrical activity } \\
\mathrm{VT} / \mathrm{VF}= & \text { ventricular tachycardia/ventricular } \\
& \text { fibrillation }
\end{aligned}
\end{aligned}
$$

These techniques have been referred to as extracorporeal life support, ${ }^{3,5}$ extracorporeal membrane oxygenation, ${ }^{6,7}$ or cardiopulmonary bypass support. ${ }^{8,9}$

In principle, prompt application of CPS could rescue patients with cardiovascular collapse, especially if reversible causes are present ${ }^{10,11}$ and neurologic function is preserved. ${ }^{12}$ In practice, however, widespread application has been limited.

In 1986, we began a program with a prospective registry of nurse-supported physician initiation of percutaneous CPS that did not initially require a perfusionist. This report reviews our experience with the urgent use of CPS in 150 patients over a 20 -year period.

\section{MATERIALS AND METHODS}

This review of patients started on emergency portable CPS at Sharp Memorial Hospital between June 1986 and December 2008 was approved by the Investigational Review Board at Sharp Healthcare.

Patients were selected by the resuscitating physician for emergency CPS when cardiac arrest persisted despite initial advanced cardiac life support or for treatment of refractory shock with imminent cardiac arrest. Patients with CPS support for elective percutaneous cardiac intervention, after a cardiotomy and after failure to wean from conventional bypass, or transferred to our institution after initiation elsewhere were excluded.

Initiation of CPS was accomplished by means of either percutaneous or cut-down placement of cannulas in the femoral or jugular vein $(18 \mathrm{~F}-20 \mathrm{~F})$ and femoral artery $(16 \mathrm{~F}-18 \mathrm{~F})$ by using methods previously described. ${ }^{13}$ If femoral artery cannulation was associated with distal limb ischemia, then antegrade perfusion was initiated by using methods previously described. ${ }^{14} \mathrm{~Pa}-$ tients undergoing recent cardiac surgery had cannulas placed in central vessels after urgent bedside sternotomy. Treatment was with a BARD (C. R. Bard, Inc, Murrayhill, NJ) hollow-fiber oxygenator in patients 1 to 57 and a heparin-bonded oxygenator (Maxima or Affinity; Medtronic, Minneapolis, Minn) in patients 58 to 150. A Biomedicus (Medtronic, Anaheim, Calif; see Figure E1) centrifugal pump was used in all cases. Before the use of heparin-bonded oxygenators and circuits, activated clotting time was maintained at approximately 300 seconds. With a heparin-bonded oxygenator and circuit, heparin was titrated to an activated clotting time of between 150 and 180 seconds or 120 and 150 seconds if bleeding ensued. Radiographic fluoroscopic guidance was used if immediately available.

Critical care nurses trained in initiation of emergency CPS were responsible for initiation of flow after priming a preassembled centrifugal pump and oxygenator circuit with Isolyte S electrolyte solution (Braun Medical, Irvine, Calif), which is composed of sodium chloride, sodium gluconate, sodium acetate trihydrate, potassium chloride, and magnesium chloride hexahydrate. When cannulation was complete, bypass tubing was connected to the arterial and venous cannulas, and flow was optimized by adjusting the centrifugal pump rotation rate.
Surgical or percutaneous interventions were undertaken if the patient appeared to have a survivable illness and if a remediable cause for cardiac arrest was present. Support was continued until a definitive corrective procedure could be performed, the patient died during bypass, or cardiovascular stabilization permitted weaning from CPS. When possible, weaning from CPS was attempted with gradual reduction of pump flow if mean arterial pressure remained greater than $60 \mathrm{~mm} \mathrm{Hg}$ on low-dose inotropic support. Most patients required transfusion of packed erythrocytes shortly after initiation of CPS.

\section{Clinical Data}

The national mortality database was queried to obtain long-term dates of death. Demographic information, preclinical conditions, support type, and cause of death were recorded in a prospective registry. Time of CPS initiation was considered routine if between 9 AM and 5 PM Monday through Friday and off hours otherwise. Cardiac rhythms at the time of cardiac arrest and refractory shock were reviewed from computerized hospital records available from the last 8 years. Cardiac causes were specified as coronary (with a history of coronary artery disease), noncoronary, or unknown cardiac. Other causes were specified as either noncardiac or unknown.

\section{Statistical Analysis}

Results are expressed as the mean \pm standard deviation. Comparisons were made with a 2-tailed Student $t$ test for continuous variables and Pearson's $\chi^{2}$ analysis for categoric data. Multivariate logistic regression was performed for long-term survival with SPSS software using variables of sex, age, cause of initiation, time of day, cause of cardiovascular collapse, and location of CPS initiation. From this, a C (convergence) statistic was derived from a receiver operating characteristic curve analysis to assess the predictive accuracy of the regression model. Kaplan-Meier analysis for time to death from the date of long-term survival $(\geq 30$ days after initiation) was performed.

\section{RESULTS}

One hundred-fifty patients (103 male and 47 female patients) aged $57 \pm 17$ years were started on emergency CPS. CPS was initiated for cardiac arrest in 127 patients (124 witnessed and 3 unwitnessed) and refractory shock in 23 patients (including 2 after defibrillation for ventricular fibrillation without CPR). Cannulation was percutaneous in 94 , cut-down in 16, open-chest in 30 (after recent cardiac surgery), and not reported in 10 patients (Table 1). Causes of cardiovascular collapse are shown in Table 2.

Overall, $39(26.0 \%)$ patients were long-term survivors (LTS; alive $\geq 30$ days with successful weaning), 30 $(20.0 \%)$ patients were short-term survivors (alive $<30$ days with successful weaning), and 81 (54\%) patients died on CPS (Figure 1). Long-term survivors had a subsequent Kaplan-Meier median survival of 9.5 years (Figure 2). In patients who were successfully weaned from CPS, 39 $(59.4 \%)$ of 69 were LTS. Duration of CPS support was 32 \pm 38 hours for LTS and $21 \pm 38$ hours for non-LTSs.

Eleven $(47.8 \%)$ patients with refractory shock and 29 $(23.4 \%)$ patients with witnessed cardiac arrest were LTS $(P<.05$, see Figure E2). Three patients with an unwitnessed cardiac arrest died on CPS.

Of 67 patients with available rhythms during cardiac arrest, $45(67.2 \%)$ had pulseless electrical activity (PEA), 
TABLE 1. Demographics for 150 patients started on emergency cardiopulmonary support over the past 20 years

\begin{tabular}{lc}
\hline Age (y) & $57 \pm 17$ \\
Support time (h) & $24.3 \pm 38.6$ \\
Sex & \\
$\quad$ Male & 103 \\
$\quad$ Female & 47 \\
Indication for extracorporeal life support & \\
$\quad$ Cardiac arrest & 127 \\
$\quad$ Refractory shock & 23 \\
Cannulation & \\
$\quad$ Peripheral, percutaneous & 94 \\
Peripheral, cut-down & 16 \\
Open-chest & 30 \\
$\quad$ Unspecified & 10 \\
Oxygenator & \\
BARD-HF 4000 & 56 \\
Heparin-bonded Maxima & 55 \\
Heparin-bonded Affinity & 39 \\
\hline
\end{tabular}

$14(20.9 \%)$ had ventricular tachycardia/ventricular fibrillation (VT/VF), and $8(11.9 \%)$ had asystole. Twelve (26.7\%) patients with PEA, $6(42.9 \%)$ patients with VT/ $\mathrm{VF}$, and $1(12.5 \%)$ patient with asystole were LTS $(P=.31)$.

CPR times of 30 minutes or longer were associated with lower long-term survival rates (see Figure E3, $P<.05$ ). Reasons for prolongation of CPR beyond 30 minutes included intermittent need for CPR, delay in decision making regarding need for CPS, and difficulty in achieving cannulation.

In patients with CPS initiated in the cardiac catheterization laboratory, long-term survival was seen in $24(50.0 \%)$ of 48 versus $15(14.7 \%)$ of 102 with CPS initiated elsewhere than the cardiac catheterization laboratory (critical care unit and other; $P<.001$ ). In the cardiac catheterization laboratory indication for CPS showed a trend to have an effect on long-term survival. No difference in CPR times in patients undergoing arrest in the cardiac catheterization laboratory versus other locations was observed (Figure 3). Seventeen $(45.9 \%)$ of 37 patients with cardiac arrest versus $7(63.6 \%)$ of 11 patients with refractory shock who had CPS

\section{TABLE 2. Prearrest cause}

\begin{tabular}{lrcc}
\hline \multicolumn{1}{c}{ Cause } & Total & Patients achieving LTS & \% LTS \\
\hline Cardiac & 113 & 34 & 31 \\
Coronary cardiac & 86 & 27 & 31 \\
Noncoronary cardiac & $20^{*}$ & 7 & 35 \\
Unknown cardiac & 7 & 0 & 0 \\
Noncardiac & $32 \dagger$ & 4 & 12.5 \\
Unknown & 5 & 1 & 20 \\
Total & 150 & 39 & 26 \\
\hline
\end{tabular}

LTS, Long-term survival *Cardiomyopathy $(\mathrm{n}=8)$, transplant rejection $(\mathrm{n}=6)$, myocarditis $(n=2)$, and "other'" $(n=4) \dagger$ Pulmonary embolus $(n=6)$, amniotic fluid embolism $(n=4)$, aortic disease $(n=3)$, septic shock $(n=2)$, and "other" $(n=17)$.

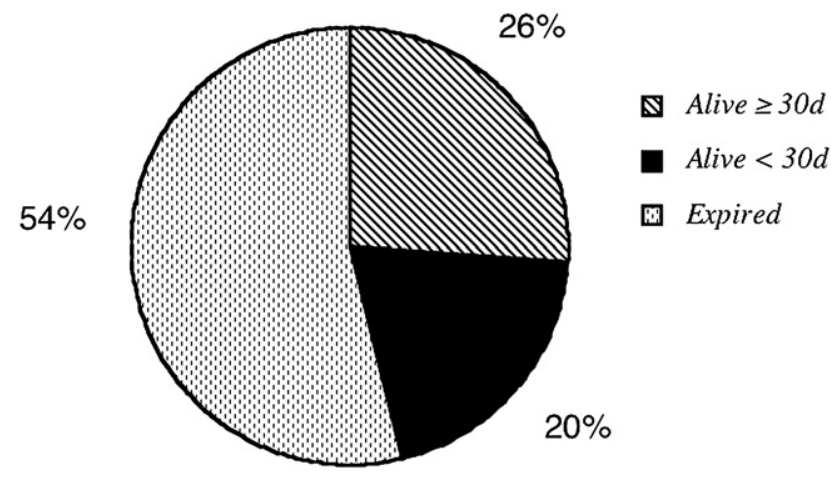

FIGURE 1. Distribution of patient survival durations. Alive $<30 d$, Weaned from cardiopulmonary support but not long-term survivors; expired, death on cardiopulmonary support.

initiated in the cardiac catheterization laboratory were LTS $(P=.77)$.

In those who died during CPS, the cause of death was persistent cardiac dysfunction in $32(39.5 \%)$ of 81 patients, whereas $23(28.4 \%)$ patients died of central nervous system (CNS)/pulmonary complications. Fifteen $(50.0 \%)$ of 30 patients who were short-term survivors (after CPS weaning) died of CNS/pulmonary causes, whereas $6(20.0 \%)$ patients died of persistent cardiac dysfunction $(P=.066$, cardiac vs CNS/pulmonary vs other).

CPS initiation time of day was available for 129 of 150 patients. Seventeen $(32.1 \%)$ of 53 patients who had CPS initiated between Monday through Friday from 9 AM to 5 PM were LTS compared with $18(24.0 \%)$ of 76 outside of these times. CPS led to left ventricular assist device implantation in $7(4.7 \%)$ patients and subsequent cardiac transplantation in $1(0.7 \%)$ of these patients. When dividing the 20-year experience in tertiles, recent recipients have had more common prearrest insertion (see Figure E4). Rates of long-term survival have not changed over time.

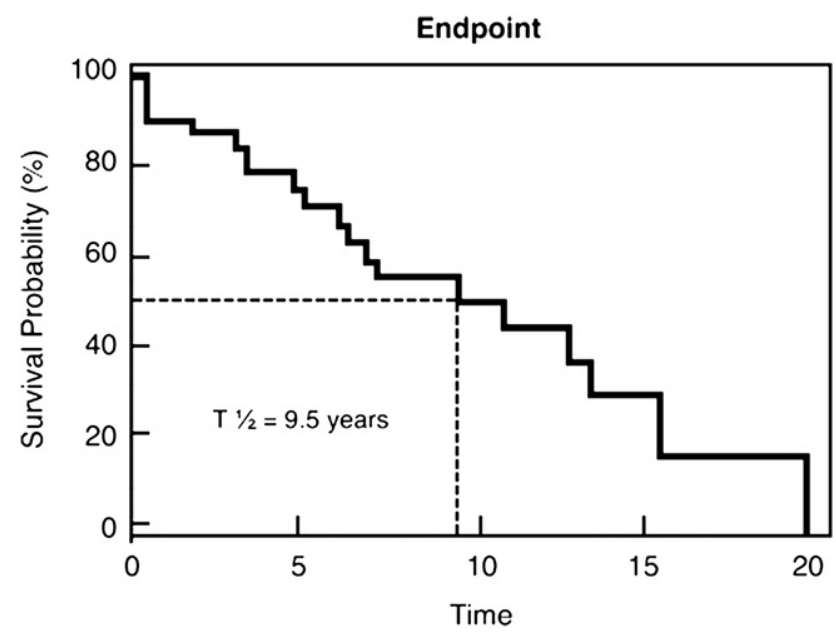

FIGURE 2. Kaplan-Meier survival in patients surviving $\geq 30$ days. $T^{1 / 2}$, Median survival. 


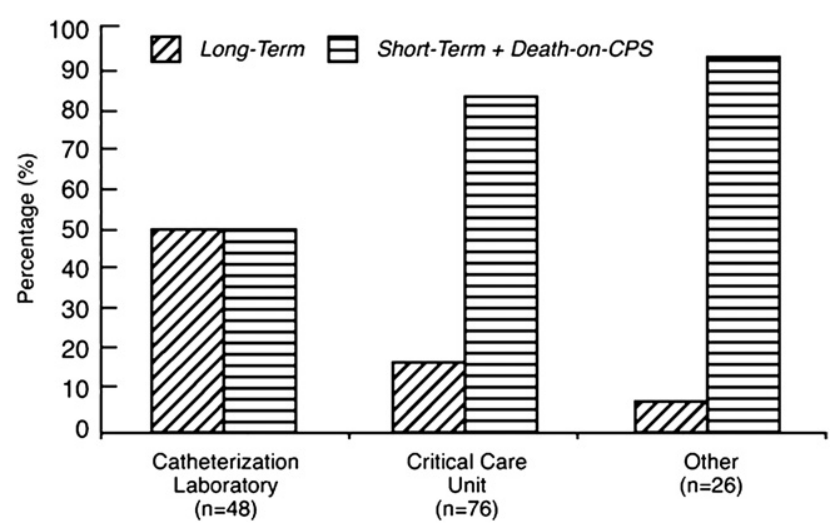

FIGURE 3. Survival by location of in-hospital cardiopulmonary support $(C P S)$ insertion.

Multivariate logistic regression of long-term survival was conducted by using variables of sex, indication for CPS initiation (refractory shock vs cardiac arrest), age, CPR time, location of CPS initiation, cause of cardiovascular collapse, and time of day. Of these values, only location of CPS initiation was found to be an independent variable predictive of outcome $(P<.01$, see Table E1). Overall, the regression model demonstrated a $\mathrm{C}$ statistic of 0.751 , indicating a modest value of predictive accuracy.

\section{DISCUSSION}

To our knowledge, this single-center report represents the largest series of consecutive cases of emergency use of CPS for cardiovascular resuscitation of patients refractory to conventional measures. Specifically excluded were patients in whom CPS was initiated for elective-procedure support, those with postcardiotomy failure to be weaned from bypass, and those transferred from an outside hospital. Overall, $26.0 \%$ of patients started on CPS were LTS, with a subsequent median survival of 9.5 years. We found the longterm survival rates for refractory shock versus witnessed cardiac arrest to be $47.8 \%$ and $23.4 \%$, respectively.

\section{Indication for CPS}

When applied for refractory shock, Combes and colleagues $^{7}$ reported a CPS-associated 28-day survival rate of $48 \%$. In patients with persistent cardiac arrest, Chen and associates $^{3}$ retrospectively compared outcomes in patients with longer than 10 minutes of conventional CPR who subsequently underwent either CPS $(\mathrm{n}=59)$ or continued conventional CPR $(n=113)$. Using a propensity score to match potential prognostic factors, there was a significant difference in 30-day survival (hazard ratio, 0.47; 95\% confidence interval, $0.28-0.77 ; P=.003$ ) and 1-year survival (hazard ratio, $0.53 ; 95 \%$ confidence interval, $0.33-0.83 ; P<.006){ }^{7}$ favoring extracorporeal CPS over conventional CPR. CPS 30-day survival was $33.9 \%$, and 1 -year survival was $18.6 \%{ }^{3}$ Thus CPS is associated with about a $25 \%$ long-term survival. Fifty percent of those weaned from CPS are alive 30 days after initiation of support.

Multiple factors could contribute to improved outcomes in the cardiac catheterization laboratory. The cardiac catheterization laboratory environment provides fluoroscopy and availability of adjunctive support equipment. Physician and support staff are present. Alternatively, the cardiac catheterization laboratory site could be a marker for patients with reversible pathologies or better prognoses. There might be less reluctance to initiate CPS support earlier on the part of the physician given the above considerations. No difference in CPR times in patients undergoing arrest in the cardiac catheterization laboratory versus other locations was observed. In a recent report from a multicenter registry of CPS applied for cardiovascular collapse, survival to hospital discharge was $27 \%$. Although initiation of support was not specified, percutaneous cannulation technique was one of the several independent variables predicting survival in a multivariate analysis. ${ }^{15}$

It is uncertain how to replicate outcomes in the cardiac catheterization laboratory for patients in critical care units or other environments. CPS placement could be initiated sooner in patients with rapidly progressive shock before cardiac arrest. Alternatively, patients could be either transported to a cardiac catheterization laboratory environment, or crucial aspects of the cardiac catheterization laboratory environment could be replicated in intensive care units.

Our data suggest that the likelihood for long-term survival with cardiac arrest might be influenced by the associated cardiac rhythm during a cardiac arrest. Although only a trend, patients with VT/VF had long-term survival of $42.9 \%$ versus $25 \%$ in patients with PEA and $12.5 \%$ in patients with asystole. In animal models of ventricular fibrillation, application of CPS improves the subsequent likelihood of cardioversion and survival compared with continued precordial compression and external defibrillation. ${ }^{16,17} \mathrm{~A}$ recent report in 11 patients found that CPS permitted termination of previously refractory ventricular tachycardia. ${ }^{18}$

In the event of cardiac arrest requiring CPR, the decision to use CPS must be prompt because survival is unlikely when CPR extends beyond 30 minutes. Factors that influence the decision to institute CPS should include whether the patient has a remediable underlying disease process. Previous studies support a strategy of CPS as a bridge to implantable left ventricular assist device or heart transplantation in adult patients. ${ }^{11,19,20}$ However, only a small portion of our patient population was bridged to other forms of cardiac replacement.

\section{Cause of Death}

In general, after CPS, subsequent death from cardiac causes implies an initial irreversible cardiovascular pathology associated with cardiovascular collapse, whereas death of CNS/ 
pulmonary causes suggests that CPS was either insufficient or delayed to maintain extracardiac oxygenated blood flow to vital organs. Thus it is not surprising that the primary cause of death in patients who were not weaned from CPS was cardiac. In contrast, among patients who were successfully weaned but were not LTS, cardiac complications were overcome, but neurologic/pulmonary complications were not.

\section{LIMITATIONS}

Conclusions regarding the efficacy of CPS for emergency resuscitation in our series are limited by the lack of a control group. As noted above, although a case-control series with matched patients from a cardiac arrest registry has been reported, ${ }^{3}$ a randomized trial would be difficult to achieve. As instrumentation and techniques continue to evolve, registry data might have to suffice for evaluation of the efficacy of CPS for cardiovascular collapse. A uniform patient selection criterion for initiating CPS was not prespecified in this series; however, this allowed assessment in a range of patients more diverse than previous studies. This is a single-center study that cannot be replicated in all centers; it still represents the largest series reported to date. Detailed complication-related data were not acquired for this registry.

\section{IMPLICATIONS FOR FUTURE IMPROVEMENTS}

Rapid application of CPS can temporarily support patients with cardiovascular collapse and permit an assessment of potential options for long-term survival. Before initiation, the likelihood of long-term survival should be considered based on the reversibility of the cause of collapse, the expected duration of CPR, and patient location within the hospital. Translating the results of CPS resuscitation in the cardiac catheterization laboratory to other hospital environments could further improve outcomes for the still-challenging condition of cardiovascular collapse.

\section{References}

1. Cummins RO, Chamberlain D, Hazinski MF, et al. Recommended guidelines for reviewing, reporting, and conducting research on in-hospital resuscitation: the inhospital "Utstein style." American Heart Association. Circulation. 1997;95: 2213-39.
2. Peberdy MA, Kaye W, Ornato JP, et al. Cardiopulmonary resuscitation of adults in the hospital: a report of 14720 cardiac arrests from the National Registry of Cardiopulmonary Resuscitation. Resuscitation. 2003;58:297-308.

3. Chen YS, Lin JW, Yu HY, et al. Cardiopulmonary resuscitation with assisted extracorporeal life-support versus conventional cardiopulmonary resuscitation in adults with in-hospital cardiac arrest: an observational study and propensity analysis. Lancet. 2008;372:554-61.

4. Gibbon JH Jr. Application of a mechanical heart and lung apparatus to cardiac surgery. Minn Med. 1954;37:171-85.

5. Dembitsky WP, Moreno-Cabral RJ, Adamson RM, Daily PO. Emergency resuscitation using portable extracorporeal membrane oxygenation. Ann Thorac Surg. 1993;55:304-9.

6. Muller E, Munch K. Servoregulation of centrifugal pumps. A new technical approach to improve patient safety during long-term extracorporeal life support. ASAIO J. 1996;42:282-7.

7. Combes A, Leprince P, Luyt CE, et al. Outcomes and long-term quality-of-life of patients supported by extracorporeal membrane oxygenation for refractory cardiogenic shock. Crit Care Med. 2008;36:1404-11.

8. Shawl FA, Domanski MJ, Wish MH, Davis M. Percutaneous cardiopulmonary bypass support in the catheterization laboratory: technique and complications. Am Heart J. 1990;120:195-203.

9. Saeed D, Kizner L, Arusoglu L, et al. Prolonged transcutaneous cardiopulmonary support for postcardiotomy cardiogenic shock. ASAIO J. 2007;53:e1-e3.

10. Smedira NG, Moazami N, Golding CM, et al. Clinical experience with 202 adults receiving extracorporeal membrane oxygenation for cardiac failure: survival at five years. J Thorac Cardiovasc Surg. 2001;122:92-102.

11. Willms DC, Atkins PJ, Dembitsky WP, Jaski BE, Gocka I. Analysis of clinical trends in a program of emergent ECLS for cardiovascular collapse. ASAIO J. 1997;43:65-8.

12. Massetti M, Tasle M, Le PO, et al. Back from irreversibility: extracorporeal life support for prolonged cardiac arrest. Ann Thorac Surg. 2005;79:178-83.

13. Jaski BE, Lingle RJ, Overlie $P$, et al. Long-term survival with use of percutaneous extracorporeal life support in patients presenting with acute myocardial infarction and cardiovascular collapse. ASAIO J. 1999;45:615-8.

14. Jaski B, McClendon $\mathrm{P}$, Branch $\mathrm{K}$, et al. Anterograde perfusion in acute limb ischemia secondary to vascular occlusive cardiopulmonary support. Catheter Cardiovasc Diagn. 1995;35:373-6.

15. Thiagarajan R, Brogan T, Scheurer M, et al. Extracorporeal membrane oxygenation to support cardiopulmonary resuscitation in adults. Ann Thorac Surg. 2009 87:778-85.

16. Gazmuri RJ, Weil MH, von PM, Gazmuri RR, Shah DM, Rackow EC. Cardiac resuscitation by extracorporeal circulation after failure of conventional CPR. $J$ Lab Clin Med. 1991;118:65-73.

17. Levine R, Gorayeb M, Safar P, Abramson N, Stezoski W, Kelsey S. Cardiopulmonary bypass after cardiac arrest and prolonged closed-chest CPR in dogs. Ann Emerg Med. 1987;16:620-7.

18. Tsai FC, Wang YC, Huang YK, et al. Extracorporeal life support to terminate refractory ventricular tachycardia. Crit Care Med. 2007;35:1673-6.

19. Bowen FW, Carboni AF, O'Hara ML, et al. Application of "double bridge mechanical" resuscitation for profound cardiogenic shock leading to cardiac transplantation. Ann Thorac Surg. 2001;72:86-90.

20. Chen YS, Ko WJ, Lin FY, et al. Preliminary result of an algorithm to select proper ventricular assist devices for high-risk patients with extracorporeal membrane oxygenation support. J Heart Lung Transplant. 2001;20:850-7. 


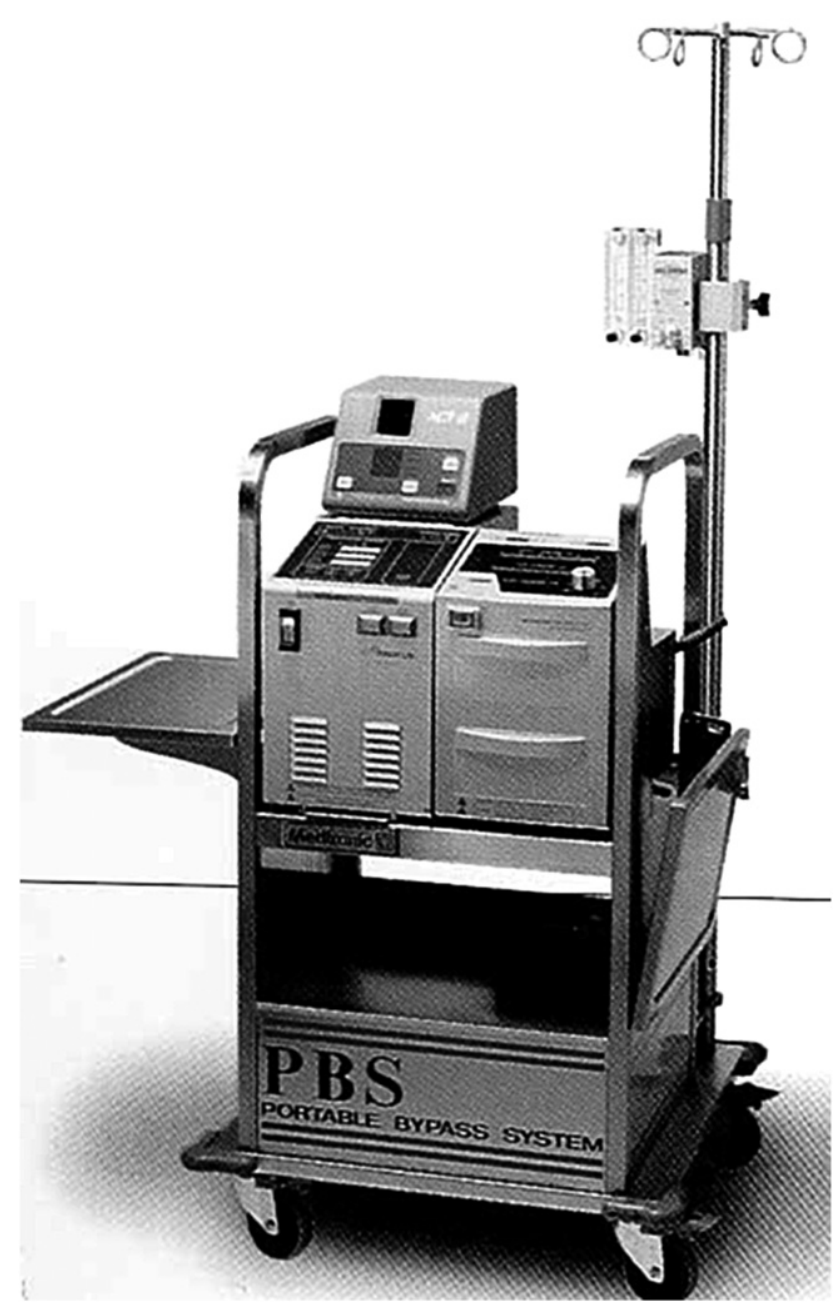

FIGURE E1. Cardiopulmonary support cart.

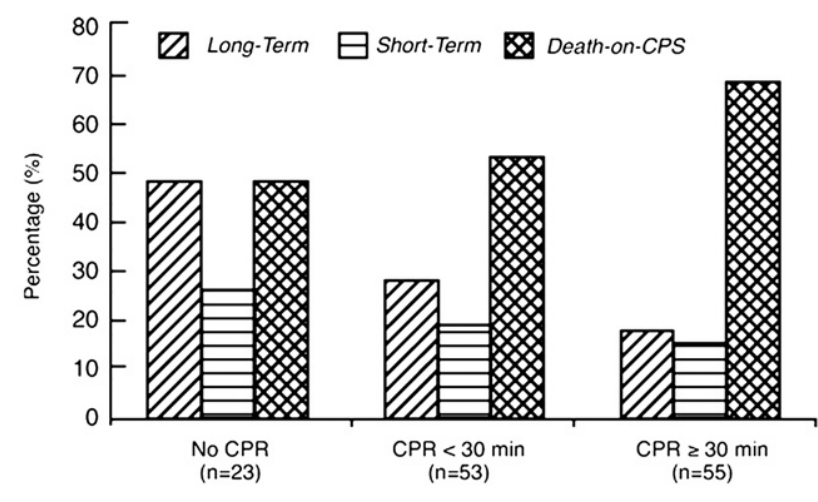

$\begin{array}{llll}\text { Long-term } & 47.8 & 28.3 & 18.2 \\ \text { Short-term } & 26.1 & 18.9 & 14.5 \\ \text { Death-on-CPS } & 26.1 & 52.8 & 67.3\end{array}$

FIGURE E3. Outcomes ofcardiopulmonary resuscitation $(C P R)$ time. CPS, Cardiopulmonary support.

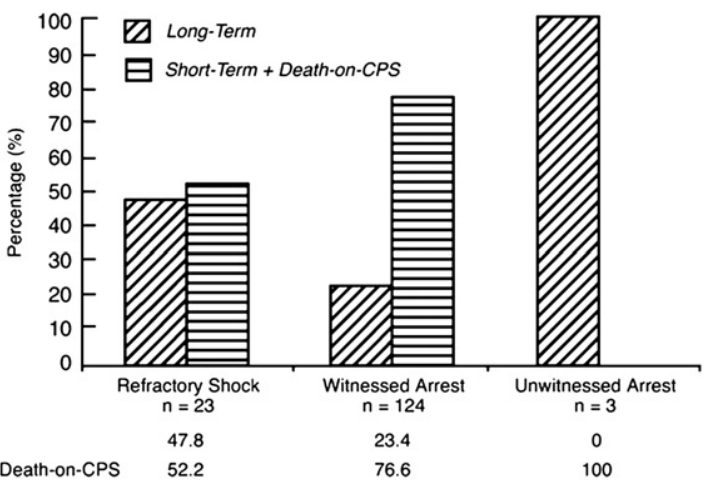

FIGURE E2. Cause of initiation of cardiopulmonary support (CPS).

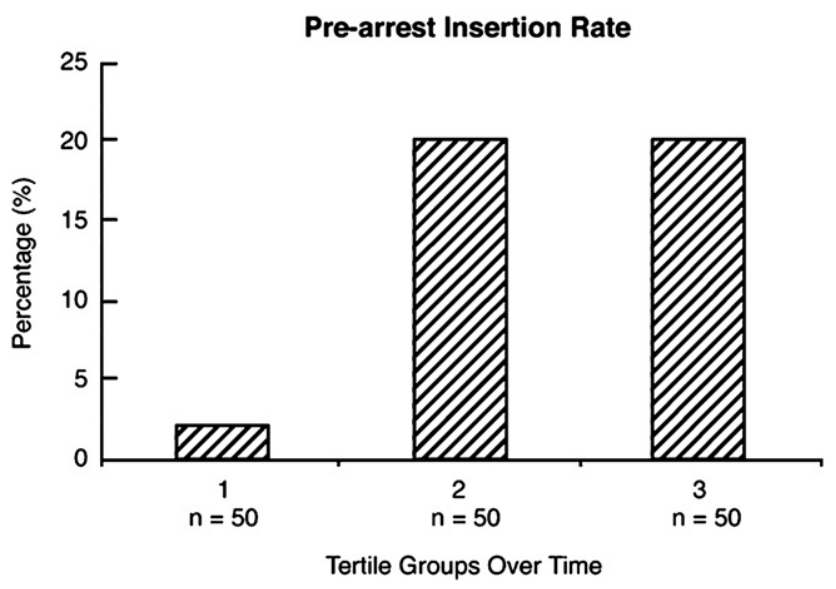

FIGURE E4. Prearrest insertion rate. 
TABLE E1. Multivariate analysis

\begin{tabular}{|c|c|c|c|c|c|c|}
\hline Variables & $\begin{array}{l}\text { Dummy-coded } \\
\text { vectors }\end{array}$ & B & SE & $P$ value & OR & $95 \% \mathrm{CI}$ \\
\hline \multirow[t]{3}{*}{ Location } & & & & .007 & & \\
\hline & Critical care unit & 1.36 & 0.50 & .007 & 3.90 & $1.45-10.44$ \\
\hline & ED/floor/other & 2.37 & 0.92 & .010 & 10.65 & $1.76-64.27$ \\
\hline Sex & & -0.25 & 0.49 & .600 & 0.78 & $0.30-2.01$ \\
\hline Arrest & & 0.24 & 1.55 & .877 & 1.27 & $0.06-26.46$ \\
\hline \multirow[t]{4}{*}{ CPR time } & & & & .588 & & \\
\hline & $\mathrm{CPR} \leq 29 \mathrm{~min}$ & 0.21 & 1.49 & .887 & 1.24 & $0.07-22.81$ \\
\hline & $\mathrm{CPR} \geq 30 \mathrm{~min}$ & 0.49 & 1.53 & .751 & 1.63 & $0.08-32.70$ \\
\hline & Unknown & -0.51 & 1.64 & .755 & 0.60 & $0.02-14.96$ \\
\hline Age & & 0.02 & 0.01 & .304 & 1.02 & $0.99-1.04$ \\
\hline \multirow{3}{*}{$\begin{array}{l}\text { Time/day for } \\
\text { implantation }\end{array}$} & & & & .792 & & \\
\hline & Weekday (9 Ам-5 Рм) & 0.28 & 0.50 & .579 & 1.32 & $0.50-3.47$ \\
\hline & Unknown & -0.12 & 0.65 & .849 & 0.88 & $0.25-3.16$ \\
\hline \multirow[t]{5}{*}{ Cause } & & & & .655 & & \\
\hline & Cardiac noncoronary & -0.09 & 0.68 & .891 & 0.91 & $0.24-3.47$ \\
\hline & Cardiac unknown & 1.45 & 1.31 & .270 & 4.25 & $0.33-55.66$ \\
\hline & Noncardiac & 0.64 & 0.69 & .355 & 1.89 & $0.49-7.32$ \\
\hline & Unknown & -0.60 & 1.40 & .669 & 0.55 & $0.04-8.56$ \\
\hline
\end{tabular}

Note: For dummy-coded vectors, the reference groups are as follows (in bold): location (cardiac catheterization laboratory); CPR time (no CPR); time/day for implantation (weekend or 5 PM to 9 AM); and cause (cardiac coronary). B, Logit; SE, standard error; $O R$, odds ratio; $C I$, confidence interval. 